\title{
On experimental censorship of particle hops in bed-load transport
}

\author{
Francesco Ballio ${ }^{1}$, Siobhan L. Fathel ${ }^{2}$, David Jon Furbish ${ }^{2}$, and Alessio Radice, ${ }^{1, *}$ \\ ${ }^{1}$ Politecnico di Milano, Milan, Italy \\ ${ }^{2}$ Vanderbilt University, Nashville, Tennessee, US
}

\begin{abstract}
Observation of incomplete particle hops in imaging-based experiments with bed-load transport does not just occur with hops that are longer than an area of observation. Even short hops can be in fact incompletely measured if they cross the boundaries of the focus area. Experimental censorship biases the statistical distribution and moments of bed-load particle hops. This paper presents: a theoretical quantification of an expected effect of experimental censorship; based on this quantification, a method to correct the statistical distribution of hop lengths; an application of the method to experimental data. Applying the correction leads to significantly different values of the mean hop length, compensating the biasing effect of censorship. By contrast, nothing can be done for experimental truncation that is associated with hops longer than an applied area of observation. Experimental truncation must be avoided a priori, by appropriate design of the measurement method.
\end{abstract}

\section{Introduction}

Bed-load sediment motion can be analyzed from an Eulerian viewpoint (referred to the properties of the process at some control locations, [1, 2]) or taking a Lagrangian approach (following individual particles as they move, $[3,4]$ ). Key quantities under consideration in the latter case are the characteristics (length, duration and mean velocity) of particle motions from entrainment to disentrainment, frequently named as particle hops. Parameterizing the particle hop properties as functions of the hydro-dynamic flow has been the object of extensive investigation during the last decades ([5-10]).

Experimental measurement of particle motions is unavoidably related with a field of view, that can be represented by a river reach under investigation (for example, in field studies on transport of tracer pebbles, e.g., [11]) or by an area of observation within a laboratory flume ([12-14]). Even though the two kinds of experimentation have very different size, they share similar shortcomings in relation to the possibility to perform Lagrangian measurements. We focus on the more numerous laboratory studies. Potentialities and affordability of video imaging techniques have made them very popular for the analysis of sediment transport (e.g., $[1,3-4,8-10,12-13])$, making large data sets available for physical interpretation of the process. However, a Lagrangian observation would be ideally required to be totally independent of a field of view. By contrast, many hops may be only incompletely observed:

\footnotetext{
*Corresponding author: alessio.radice@polimi.it
} 
hops longer than the area of observation cannot be measured, this effect being called truncation in the present manuscript; hops shorter than the focus area but crossing its boundaries will also be incomplete, this effect being called censorship. Measured probability distributions and statistical moments of the hop properties can be thus biased by the occurrence of incomplete hops ([12]). It is again remarked that the effect of a finite area of observation involves censorship of all hop lengths (even the shortest in a population) and not only truncation of hops longer than the focus area, because particle entrainment events can occur close to the boundaries of the measuring window.

The paper discusses the expected effects of hop censorship. Then, experimental censorship is demonstrated with reference to recent data. A bias to experimental data may be avoided by appropriate experimental strategies or finding post-processing means to correct the statistics of hop properties by removing the effect of experimental censorship. Removing censorship of particle hops can significantly change the values obtained for the statistical moments of the key quantities.

\section{Concept}

We consider an observation of a statistically stationary and uniform bed-load process. Particle hops are assumed to have all the same length $L_{H}$, with starting points uniformly distributed in space and time. For the sake of simplicity, we initially assume that the observation is infinite in time. By contrast, an area of observation is employed with a streamwise length $L_{A}$. It must be guaranteed that $L_{A}>L_{H}$ because, otherwise, the hops will be truncated and will not be observable. Now, let one assume that a finite sample of particles travels along the system and that $N$ entrainment events take place within the area $A$. This would ideally enable $N$ hops to be observed. However, this will not be the case, because hops starting close to the downstream boundary of the area (at a distance shorter than $L_{H}$ ) will not be completely observed. The number of hops that will be completely observed is:

$$
N_{o b s}=N \times\left(1-L_{H} / L_{A}\right)
$$

based on an assumption of starting points uniformly distributed in space. The occurrence of hop censorship is depicted in Fig. 1.

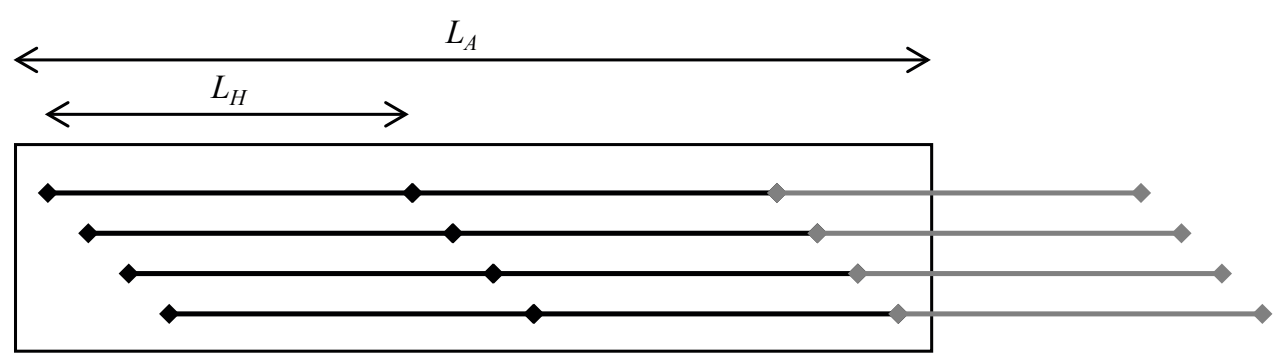

Fig. 1. Schematic diagram showing the occurrence of spatial censorship for uniform bed-load particle hops with length $L_{H}$. Motion is rightwards and the rectangle defines an observation area with length $L_{A}$. Black hops (lines bounded by diamonds) will be completely observed while grey hops will be incompletely observed.

It is well known that particle hops do not have the same length, as bed-load transport is a stochastic process. To adapt the above concept to a more realistic situation, one can apply eq. (1) to the observed hops for any possible length. In other words, $N_{i}\left(L_{H i}\right)$ is now intended as the number of hops with a certain length $L_{H i}$ starting within $L_{A}$. The combination of $L_{H i}$ 
and $N_{i}$ defines a distribution of hops starting within $L_{A}$. However, for a certain hop length $L_{H i}$, one will observe a number of hops:

$$
N_{\text {obsi }}=N_{i} \times\left(1-L_{H i} / L_{A}\right)
$$

due to spatial censorship. The distribution of observed hop lengths will be, therefore, different from the distribution of all the hop lengths for particle entrainment within $L_{A}$. This is qualitatively depicted in Fig. 2, where the occurrence of hop truncation is also retrieved, assuming that there will be also some hops with $L_{H i}>L_{A}$. Truncation, that is the impossibility to observe particle hops longer than an area of observation, takes place in the right part of Fig. 2, while censorship, that is the impossibility to observe particle hops shorter than $L_{A}$ if they start close to the boundaries of the area, is related with the left part of Fig. 2. The quantitative impact of censorship varies with $L_{H i}$, being negligible for very short hops and increasing up to $100 \%$ for $L_{H i}=L_{A}$.

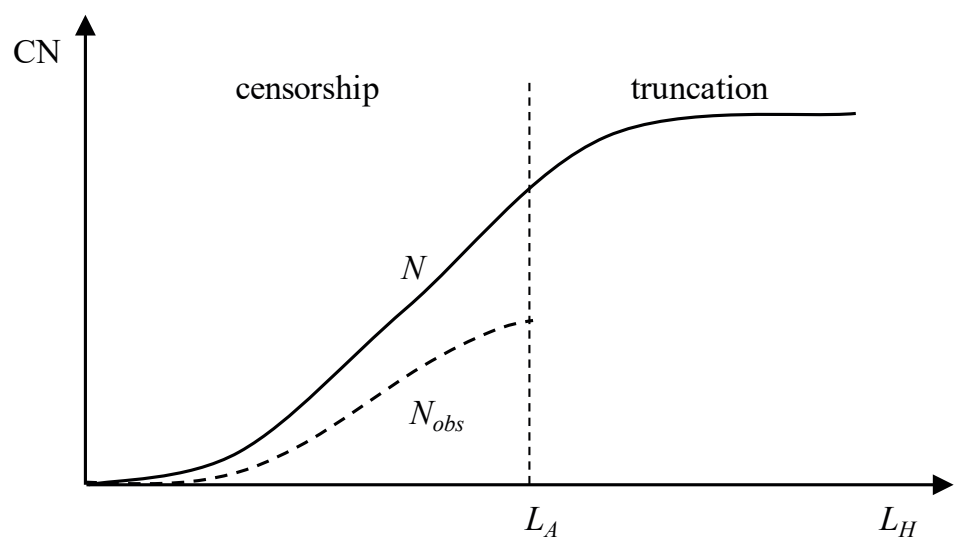

Fig. 2. Schematic diagram showing the occurrence of truncation and censorship, and how they affect the Cumulative Number $(\mathrm{CN})$ of observed hops (dashed line) compared to the real number of hops (continuous line).

What is proposed in this manuscript is that, if one can quantify the $N_{o b s i} / N_{i}$ ratio as equal to $1-L_{H i} / L_{A}$, then the latter quantity can be used as a correction factor to reconstruct a real distribution of hop lengths starting from an observed one. In the following, this correction will be applied to the Cumulative Number $(\mathrm{CN})$ and the Probability Density Function (PDF) of particle hops in a bed-load transport experiment.

\section{Application to experimental data}

We now consider the particle hop data of [14]. The experiment was performed in a pressurized duct with a cross section of $0.40 \times 0.11 \mathrm{~m}^{2}$. A fixed, rough bed was employed, which was made of glued Polybutylene-Terephthalate particles with a density of $1.27 \mathrm{~g} / \mathrm{cm}^{3}$ and a size of $3 \mathrm{~mm}$. The bulk flow velocity was equal to $0.32 \mathrm{~m} / \mathrm{s}$ and corresponded to 1.4 times a threshold flow velocity for sediment transport. Bed-load particles were fed at the upstream inlet of the flume. The motion of the transported particles was recorded from the top using a CCD camera with a frame rate of $32 \mathrm{fps}$. The area of observation was $270 \mathrm{~mm}$ in the stream-wise direction and $200 \mathrm{~mm}$ in the transverse direction. The duration of the movie was $50 \mathrm{~s}$. The tracks of the bed-load particles were obtained by Particle Tracking Velocimetry using the Streams package (http://www.civil.canterbury.ac.nz/streams.shtml), followed by a 
manual repairing of tracking errors. After the tracking procedure, 321 particle tracks were obtained, which in turn furnished 680 hops, 240 of which were complete. The statistical distribution of the hop length is the key object of investigation in this manuscript.

The $\mathrm{CN}$ of the hop length is depicted in Fig. 3. We first consider the distribution obtained from the hops that were completely measured. This distribution obviously reaches a maximum $\mathrm{CN}$ value of 240 . The tail of the distribution arrives at $231 \mathrm{~mm}$, corresponding to $86 \%$ of the stream-wise size of the observation area. A significant effect of censorship shall then be expected for this experiment (furthermore, it was reported in [14] that the PDF for all the measured hops - including also those that were incompletely observed - presented a peak at $270 \mathrm{~mm}$, demonstrating a major occurrence of track truncation). If the proposed correction is applied to the number of observed hops, a new curve is obtained that presents a different shape and arrives at a maximum $\mathrm{CN}$ value of 324.7, thus one obtains a non-integer value that must be interpreted as a statistical approximation. Furthermore, it is evident that the weight of the correction increases for larger $L_{H i}$, because for the smallest $L_{H i}$ values the correction factor is negligibly less than 1 .

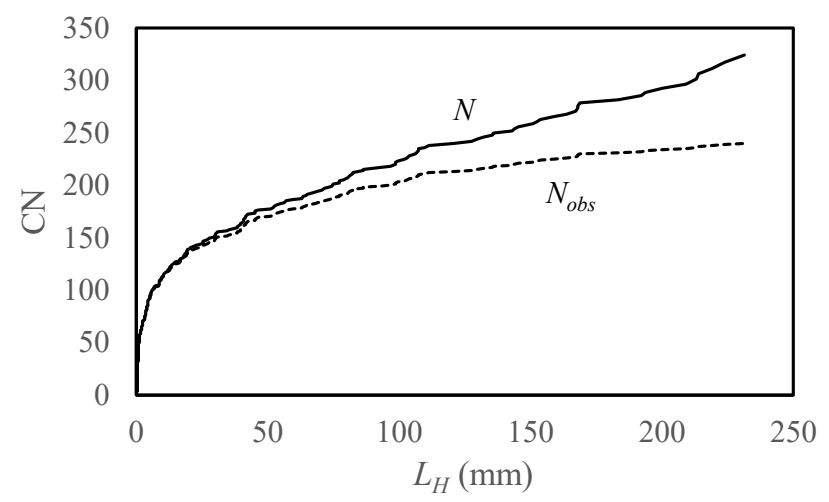

Fig. 3. Cumulative Number $(\mathrm{CN})$ of particle hop length: observed (dashed) and corrected (continuous) numbers for the data of [14].

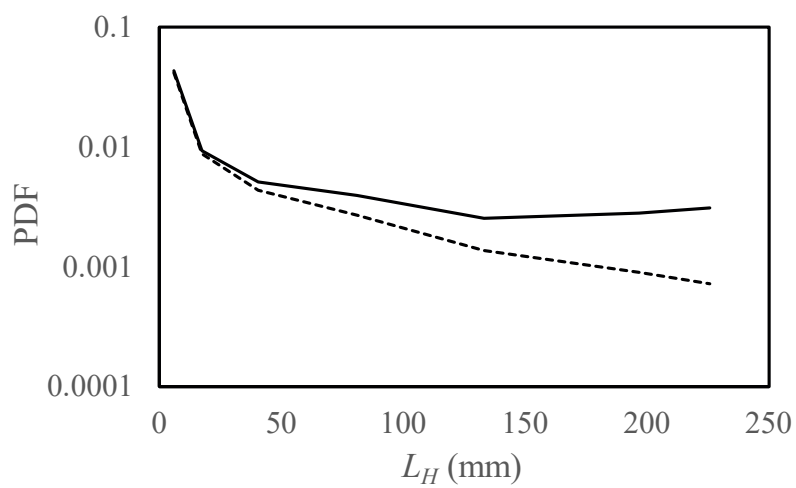

Fig. 4. Probability Density Function (PDF) of particle hop length: measured (dashed) and corrected (continuous) distributions for the data of [14].

The same approach is applied to the PDF of observed hop lengths in Fig. 4. The shape of the PDF was quite irregular due to a relatively limited sample size and was thus smoothed by an appropriate choice of bins, to make the observation of the diagram easier. Again, the probability of the shortest hops is negligibly modified by the correction, while the importance of the correction increases for increasing hop length, and the tail of the distribution is 
appreciably modified by the correction factor. The change of the shape of the PDF changes, in turn, the mean value of the hop length, that increases from $41 \mathrm{~mm}$ to $79 \mathrm{~mm}$ applying the correction (with a $93 \%$ increase).

\section{Discussion}

Experimental data used for analysis and interpretation of natural phenomena need to be as accurate as possible. On the other hand, experimental truncation and censorship may bias the probability distributions obtained for bed-load particle hops, thus flawing phenomenological interpretation by unreliable data. Censorship cannot be avoided, but the bias it induces can be corrected after the measurements. The correction proposed in this manuscript is very important for future analyses of weak bed-load processes considering the particle hops.

The proposed correction only accounts for censorship of particle hops (corresponding to hops that are shorter than an observation area but are not observed completely because they intersect the boundaries of the area), whereas nothing can be done for truncation (that corresponds to hops longer than the observation area). In the presence of a significant truncation of hop data, the correction may not return a good value. In this respect, [14] proposed to compute a mean hop length by pooling all the observed tracks together and dividing a total length by the number of entrainment events observed. For the same experiment used in this work, they obtained a hop length of $177 \mathrm{~mm}$, indicating that the correction of the PDF in Fig. 4 only retrieved $45 \%$ of the hop length, due to truncation.

The method proposed by [14] can be seen as more powerful than the correction introduced in this work, because it is able to cope with truncation and censorship, while the present correction only accounts for censorship. However, in the presence of suitable (i.e., not truncated) data, the present correction is able to return the entire PDF of the quantities and not only their mean value. This strongly stimulates the realization of experiments where truncation is avoided using a long observation window (e.g., $[15,16])$.

Even though conceptually sound, the correction proposed in this work is not 'validated', because a perfectly uncensored sample of particle hops will never be available. A first check can imply the comparison between the mean values obtained from corrected PDFs and those obtained from track pooling, but this requires using data without truncation, as mentioned above. Another aspect to be considered is the interplay between spatial and temporal censorship. In this paper, the duration of an experiment was considered much larger than the typical duration of particle hops. However, hop length may suffer from indirect censorship in space and vice-versa. Further work will be, however, devoted to generalizing the approach and providing more examples of its performance.

\section{Conclusions}

Experimental censorship, as a consequence of the incomplete observation of bed-load particle hops due to particle tracks crossing the boundaries of a focus area, biases data samples. The statistical distribution of, for example, hop lengths, is distorted and not representative of a real one for all the hops starting within the area of observation. Censorship affects particle hops of all lengths from the shortest to the length of the focus area. However, under a statistically stationary process, different hop lengths are differently prone to censorship.

In this paper, the length-dependent bias from experimental censorship was quantified theoretically. It was also proposed to invert the relationship between real and biased observation, to infer the former from the latter. In this way, it was possible to estimate a real probability distribution of hop lengths from a measured one. The quantitative impact of the 
proposed correction was demonstrated by an application to experimental data. The correction of censorship changed the mean hop length by almost $100 \%$.

In practice, censorship acts together with truncation, as a consequence of the use of a measuring area that is shorter of the longest hops. The effect of truncation cannot be compensated a posteriori, thus truncation must be avoided by appropriate experimental strategies.

We acknowledge financial support by the Research Executive Agency through the7th Framework Programme of the European Union (ITN-316546 'HYTECH' to FB). This work was also supported by the U.S. National Science Foundation (EAR-1226076 to DJF).

\section{References}

1. A. Radice, F. Ballio, V. Nikora, Water Resour. Res., 45 (2009)

2. T. Böhm, C. Ancey, P. Frey, J. Roboud, C. Ducottet, Phys. Rev. E, 69 (2004)

3. E. Lajeunesse, L. Malverti, F. Charru, J. Geophys. Res. - Earth Surf., 115 (2010)

4. J. Campagnol, A. Radice, F. Ballio, V. Nikora, J. Hydraul. Res., 53 (2015)

5. J.E. Abbott, J.R D. Francis, Philos. Trans., Royal Society of London, A284 (1977)

6. H.Y. Lee, I.S. Hsu, J. Hydraul. Eng., 120 (1994)

7. C. Hu, Y. Hui, J. Hydraul. Eng., 122 (1996)

8. A.N. Papanicolaou, P. Diplas, M. Balakrishnan, C.L. Dancey, J. Comput. Civ. Eng., 31 (1999)

9. J. Campagnol, A. Radice, R. Nokes, V. Bulankina, A. Lescova, F. Ballio, J. Hydraul. Res., 51 (2013)

10. J. Heyman, P. Bohorquez, C. Ancey, J. Geophys. Res. Earth Surf., 121 (2016)

11. M.A. Hassan, H. Voepel, R. Schumer, G. Parker, L. Fraccarollo, J. Geophys. Res. Earth Surf.,118 (2013)

12. S.L. Fathel, D.J. Furbish, M.W. Schmeeckle, J. Geophys. Res. - Earth Surf., 120 (2015)

13. J.C. Roseberry, M.W. Schmeeckle, D.J. Furbish, J. Geophys. Res. - Earth Surf., 117 (2012)

14. F. Ballio, D. Pokrajac, A. Radice, S.A. Hosseini Sadabadi, J. Geophys. Res. - Earth Surf., 123 (2018)

15. A. Radice, S. Sarkar, F. Ballio, J. Vis. Exp., 125 (2017)

16. M Cassel, H. Piégay, J. Lavé, Earth Surf. Proc. Land., 42 (2017), 1111 